\title{
A case study from M.D. Anderson Cancer Center: 26-year-old female with self-palpated axillary adenopathy
}

\author{
J. Oh, H. Kuerer, E. Rivera, S. Kronowitz \\ M.D. Anderson Cancer Center, Houston, TX, USA.
}

\section{Clinical presentation}

Ms. L.G. is a 26-year-old female with no family history of cancer who self-palpated a mass in her right axilla in early 2004. Bilateral diagnostic mammogram at an outside hospital revealed calcifications in the upper outer quadrant of the right breast and core needle biopsy from this quadrant revealed atypical ductal hyperplasia. She was referred to M.D. Anderson and initial physical examination was positive for a $3.5 \times$ $4.5 \mathrm{~cm}$, ill-defined mass at the upper outer quadrant of the right breast associated with a $2 \mathrm{~cm}$, mobile axillary lymph node.

\section{Objective staging data}

Complete work-up including repeat bilateral mammogram, breast and surrounding lymphatic ultrasound studies, computed tomography (CT) scan of the abdomen and pelvis, bone scan and fine needle aspiration (FNA) from the right axillary lymph mode and upper outer breast lesion were performed. The pathology from the axillary FNA was read as metastatic adenocarcinoma of breast primary, $100 \%$ estrogen receptor (ER) positive, 90\% progesterone receptor (PR) positive and human epidermal growth factor receptor (HER) 2N negative. FNA of the breast revealed necrotic debris. Internal pathology review of the outside biopsy from the breast revealed ductal carcinoma in situ (DCIS). The mammogram revealed a large area of pleomorphic calcifications spanning $11 \times 7 \times 8 \mathrm{~cm}$ centered at the right upper breast but

Correspondence to: Julia L. Oh, MD, The University of Texas M.D. Anderson Cancer Center, 1515 Holcombe Blvd, Unit 1202, Houston, TX 77030, USA. Tel: +1 713563 2300; Fax: +1 7195636940

Publication date 29/07/05

BCO/412/2005/CS no mass was seen. Breast ultrasound identified a $1.7 \times 1.2 \times 1.0$ mass at the 11 o'clock position of the right breast and at least two abnormal right axillary lymph nodes, the largest measuring $2 \times 1.3 \times 1.3 \mathrm{~cm}$. The remainder of her metastatic work-up was completely negative.

\section{Treatment and outcome}

She was discussed at our breast multidisciplinary meeting and examined by the surgery, medical oncology and radiation oncology services. She was clinically staged a TxN1M0 Stage II patient with the assumption of invasive breast disease. The patient was not a candidate for breast conservation therapy in view of her extensive calcifications but a delayedimmediate approach to reconstruction with the insertion of a tissue expander at the time of her skinsparing mastectomy was anticipated. The consensus recommendation was for systemic chemotherapy, surgery and post-mastectomy radiation therapy based on final pathology. Neoadjuvant chemotherapy was initiated with weekly paclitaxel $\times 12$ followed by 5 -flurouracil epirubicin cyclophosphamide $($ FEC) $\times 4$ cycles. Follow-up mammogram and ultrasound revealed stable calcifications in the breast and a slight decrease in axillary adenopathy, now measuring $1.4 \times 0.8 \times 0.7 \mathrm{~cm}$.

In January 2005, the patient underwent a skinsparing right mastectomy with insertion of a fully inflated tissue expander to preserve the dimensions of the breast skin envelope. Pathology revealed two foci of invasive, Grade 3 carcinoma, the larger one measuring $0.45 \mathrm{~cm}$ and both involved extensive Grade 3 DCIS spanning $10 \mathrm{~cm}$ with focal necrosis. The tumor was positive for lymphovascular invasion and Level-II axillary dissection revealed 8/15 lymph nodes containing metastatic tumor. No extracapsular extension 
was noted. She was therefore upstaged to a pT1(multicentric)N2M0 Stage IIIA and post-mastectomy radiation therapy to the right chest wall, internal mammary nodes, supraclavicular and infraclavicular regions was recommended. The tissue expander was deflated on the morning of her radiation oncology simulation and will remain deflated throughout radiotherapy. She is currently in her last week of postmastectomy radiotherapy, has expected erythema throughout the treatment fields and no complications related to the tissue expander. Gradual re-expansion and delayed autologous breast reconstruction after complete skin healing occurs is anticipated.

\section{Comments}

Ms. L.G. highlights several important points of interest. First, although uncommon, breast cancer does occur in women as young as 26 years old and prompt diagnosis and treatment are critical. Second, diffuse calcifications have been associated with a higher rate of axillary lymph node involvement and proved to be so in this case. The unique presentation of axillary metastases without a primary, combined with her young age and diffuse calcifications on mammogram should raise our level of concern for more advanced disease. This is paramount in the timing of breast reconstruction as an immediate reconstruction based on preoperative indications for adjuvant radiotherapy would have compromised both her delivery of radiotherapy and ultimate cosmetic outcome of an irradiated breast mound. Ms. L.G. is an example where individual features put her into a higher risk group than initially derived from strict clinical staging procedure. These patients require innovative approaches to optimize oncologic and aesthetic outcomes. 